\title{
Inhalt.
}

\section{Erster Abschuit.}

Allgemeines ïber sedimentäre Bil-

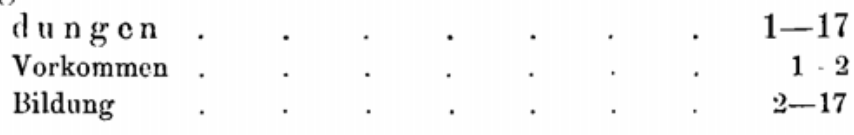

\section{Erste Abtheilung.}

Carbonatgesteine $\quad . \quad . \quad . \quad . \quad$. $18-91$

\section{Kapitel XLII.}

Sedimentäre Kalksteine, welche dem

blossen Auge amorph erscheinen 18-41

Conservation des kohlensauren Kalk im Mecr . $35-26$

Zusammensetzung der Kalksteine . . . . 26-28

Unreine Kalksteine . . . . . . 29-30

Metamorphosirte Gesteine, entstanden durch Verdrängung von Kalksteinen . . . . . 31-41

\section{Kapitel XuIV.}

Sedimentïre krystallinische Kalksteine. Körniger Kalk. Marmor. $42-51$ Vorkommen. . . . . . $42-43$ Zusammensetzung . . . . . . . . 43-44 Bildung als Absatz von Gewässern . . . 44-48 Bildung aus sedinentären amorphen Kalksteinen . 48-51 


\section{Kapitel XWV.}

Sedimentäre krystallinische KalkMagnesiagesteine. Dolomit . . 52-91 A. Geognostische Verhältnisse . . . . . 53-74 B. Genetische Verhältnisse . . . . . 74-91

\section{Zweite Abtheilung.}

Sedimentäre Silicatgestcine . . . . 92-129

Bildung

\section{Kapitel XHVI.}

Thonschiefer, Grauwacke, Schalsteine, Alaun- und Brandschiefer, Schieferthonc und Thone. . . . 93-129

A. Thonschiefer (Urthonschiefer und Petrefacten führende Thonschiefer) . . . . 93-120

Vorkommen . . . . . . . . . . . . 493

Bildung . . . . . . . . . $93-100$

Mineralogische Zusammensetzung . . . 101-103

Elementare Zusammensetzung der Thonschiefer . 103-108

Zersetzung . . . . . . . $114-120$

B. Grauwacke . . . . . . . . . . 120

Vorkommen . . . . . . . . . 120

Elementare Zusammensetzung . . . . . $\quad 120$

Mineralogische Zusammensetzung $\quad . \quad$. $\quad . \quad 120$

C. Schalsteine . . . . . . . . . . . .

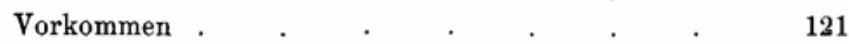

Zusammensetzung und Bildung . . . . 121-124

D. Alaun- und Brandschiefer . . . . . . . .

Zusammensetzung . . . . . . . . $125-126$

E. Schieferthone . . . . . . . . . .

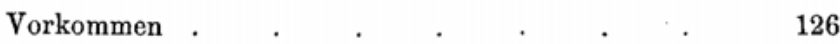

Zusammensetzung und Bildung . . . . 126-127

F. Thone . . . . . . . . . . . . . . . .

Zusammensetzung und Bildung. . . . $127-129$

\section{Dritte Abtheilung.}

Conglomerirter Detritus . . . . . . . . 


\section{Kapitel XLVII.}

Sandsteine und Con glomerate seito A. Sandsteinc . . . . . . . . . . . . 130-160

Vorkommen . . . . . . . . . . . 130

Mineralogische Zusammensetzung . . . . 130-137

Elementare Zusammensetzung . . . . 137-149

Bildung . . . . . . . . . $149-157$

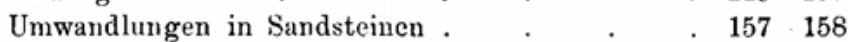

Zersetzung der Sandsteine. . . . . . 158-160

I3. Conglomerate . . . . . . . . . . . .

Vorkommen . . . . . . . . . . . 160

Zusammensetzung . . . . . . $\quad . \quad 160162$

Bildung . . . . . . . . . . . 162

\section{Zweiter Abschuit.}

Allgemeines ïber Metamorphosen sedimentärer Silicatgesteine . . .163-211

Vorkommen . . . . . 164-165

Bildung . . . . . . . . . 165-211

Kapitel XWVIII.

Metamorphische Silicatgesteinc . . .212-251

A. Glimmerreiche 'Thonsehiefer . . . .212--215

Vorkommen . . . . . . . . . 212

Mineralogische und olementare Zusammensetzung $212 \quad 215$

13. Glimmerschiefer . . . . . . . . . . . . $215-23$

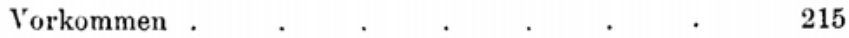

Mineralogische Znsammensetzung . . . 215-216

Elementare Zusammensetzung . . . . . 216-220

Bildung . . . . . . . . . 220-222

Unwandlung und Zersetzung . . . . 222-223

C. Chloritschiefer . . . . . . . . . . . . 235

Vorkommen . . . . . . . . . . . 223

Mineralogische Zusammensetzung . . . 223-225

Bildung . . . . . . . . . . 225-226

Umwandlung des Chloritschiefer in Glimmersehiefer 226-235

D. 'Talkschiefer $\quad . \quad$. . . . . . . . . . . .

Vorkommen . . . . . . . . . 235

Mineralogische Zusammensetzung . . . . $235-236$

Elementare Zusammensetzung . . . . 336-337

Bildung . . . . . . . . . . 237

Zersetzung . $\quad . \quad$. $\quad . \quad$. $\quad . \quad$. 237-238 
T. Serpentitgete.

E. Serpentingestein (Serpentinschiefer) . . 238

Vorkommen . . . . . . . . . . . $\quad 238$

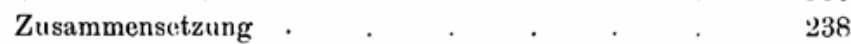

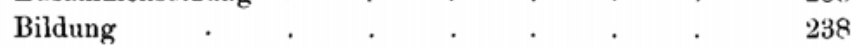

F. Gneifs . . . . . . . . . . . . . . $238-249$

Vorkommen .

Mineralogische Zusammensetzung $\quad$. $\quad$. $\quad$. $\quad 339$

Elementare Zusammensetzung . . . . . . 239-242

Bildung . . . . . . . . . . 242-245

Zersetzung . . . . . . . . . 245-249

G. Granulit . . . . . . . . . . . . . . . . 2491

Vorkommen . . . . . . . . . . 249

Mineralogische Zusammensetzung $\quad . \quad$. $\quad$. 249

Elementare Zusammensetzung . . . . . 249-251

Bildung . . . . . . . . . . 251

Umwandlung . $\quad . \quad$. $\quad . \quad . \quad . \quad . \quad . \quad 251$

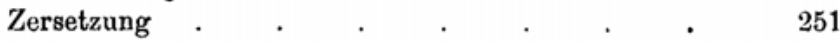

\section{Dritter Abschitt.}

Allgemeines über massige krystal-

linische Silicatgesteine. . . 252-299

Vorkommen . . . . . . . . 253

Bildung im Allgemeinen . $\quad$. . . . . 253-266

Bildung primitiver Gesteine und Bildung sedimen-

tärer Gesteine aus erodirten Theilen derselben $266 \quad 278$

Einschlüsse in krystallinischen Gesteinen . . 278-2\$9

Zersetzung . . . . . . . . 289-299

\section{Kapitel XIIX.}

Granit . . . . . . . . . . . . . .

Vorkommen . . . . . . . . . $300-301$

Mineralogische Zusammensetzung . . . 301-302

Eiementare Zusammensetzung . . . . 302-305

Bildung . . . . . . . $305-314$

Zersetzung $. \quad . \quad . \quad . \quad . \quad . \quad$. $314-321$

Kapitel I.

Felsitporphyr (quarzhaltiger und quarzfreicr). . . . . . . . . . . $322-331$

Vorkommen . . . . . . . . . 322

Mineralogische Zusammensctzung . . . . 322-323

Elementare Zusammensetzung . . . . . $323-326$ 


\begin{tabular}{|c|c|c|c|c|c|c|c|}
\hline Bildung & . & - & 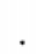 & . & - & $\cdot$ & $\begin{array}{r}\text { Seite. } \\
\text {. } 326-327\end{array}$ \\
\hline Zersetzung & . & . & . & - & $\sigma^{\circ}$ & . & . $327-331$ \\
\hline
\end{tabular}

\section{Kapitel HI.}

Perlsteine und Pechsteine. . . . .332-338

A. Perlsteine . . . . . . . . . . . .

Vorkommen . . . . . . . . 332

Elementare Zusammensetzung . . . . . 332-333

Bildung und Zersetzung . . . . . . . 333

B. Pechsteine . . . . . . . . .

Vorkommen . $\quad . \quad . \quad . \quad . \quad . \quad . \quad . \quad 333$

Mineralogische Zusammensetzung . . . . 334-335

Elementare Zusammensetzung . . . . . 335337

Zersetzung . . . . . . $337-338$

\section{Kapitel III.}

Trachytisehe Gesteine . . . . . . . .

Vorkommen . . . . . . . . 339

Mineralogische Zusammensetzung . . . . 339-341

Elementare Zusammensetzung . . . . . 341-345

Bildung . . . . . . 345-346

Zersetzung . . . . . . . . $346-355$

\section{Kapitel LIII.}

II ornblendegesteine . . . . . . . . . .

Vorkommen. A. Syenit . . . . . . 356

B. Hornblendeschiefer . $\quad . \quad$. $\quad . \quad$. 356

C. Diorit . 356

Mineralogische Zusammensetzung. A. Syenit . 356-357

B. Hornblendeschiefer . . . . . 357

C. Diorit . . . . . . . . $357-358$

Elementare Zusammensetzung . . . . 358-362

Bildung . . . . . . . . . . $\quad . \quad 363$

Zersetzung . . . . . . . . 364-365

\section{Kapitel LIV.}

Phonolith . . . . . . . . . . $366-371$

Vorkommen . . . . . . . . 366

Mineralogische Zusammensetzung . . . . 366-367

Elementare Zusammensetzung . . . . . 367-368

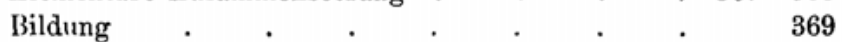

Zersetzung . $\quad . \quad . \quad . \quad . \quad . \quad . \quad 369-371$ 


\section{Kapitel LV.}

B a salt . . . . . . . . . . . . . .

Vorkommen . . . . . . . . . 372

Mineralogische Zusammensetzung . . . . $372-373$

Elementare Zusammensetzung . . . . . $\quad$. $373 \quad 376$

Bildung . . $\quad . \quad 5 \quad . \quad$. $\quad . \quad$. $376-421$

Regeneration des Basalt aus geschmolzenem Basalt 421-424

Umwandlung und Zersetzming . . . . . 424-445

\section{Kapitel LVI.}

Basaltische Gesteine . . . . . . 446-471

Vorkommen . . . . . . . . 446-447

Mineralogische Zusammensetzung. Dolerit . . 447

Melaphyr . $\quad$. $\quad . \quad$. $\quad . \quad$. $447-448$

Labrador und Augitporphyr . . . . . 448

Gabbro $\quad . \quad$. $\quad . \quad$. $\quad$. $\quad$. $\quad$. 448

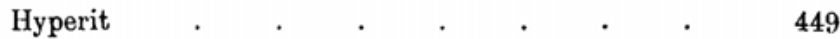

Diabas . $\quad . \quad$. $\quad . \quad$. $\quad . \quad$. $\quad . \quad 449$

Elementare Zusammensetzung . . . . . 449-455

Bildung $\quad . \quad$. $\quad . \quad . \quad . \quad . \quad . \quad 455-456$

Zersetzung der Dolerite . . . . . . 456-457

Zersetzung der Melaphyre . . . . 457-463

Zersetzung der Spilite . . . . . . . 463-464

Umwandlung und Zersetzung des Augitporphyr . 464-465

Umwandlung und Ausscheidung des Gabbro . 466

Zersetzung des Gabbro . . . . . . . 466-467

Zersetzung des Hyperit . . . . . . 4 467-468

Zersetzung des Diabas . . . . . . 469-471

\section{Vierter Abschnitt.}

\section{Kapitel LVII.}

Erdbeben und Bergschlipfe . . .472-603

Bergschlipfe. Geognostische und genetische Verhältnisse . . . . . . . .

Firdbeben. Geographische und geognostische Verhältnisse . . . . . . . . 477-486

Erdbeben und coordinirte Frscheinungen . . 486

Nasse Witterung und Erdbeben . . . . 486-489

Erweichbare und leicht bewegliche Schichten . 489

Frweichte Thonlager . . . . . . . 4 $489-491$

Vorkommen der Thonlager in sedimentären Formationen . . . . . . . . 491-492 
Erdbeben und Senkungen . . . . . 495-499

Erdbeben und Hebungen. . . . . 499-508

Erdbeben und Spaltenbildung . . . . 508-510

Erdbeben und Schlammströme . . . . 510-512

Erdbeben und Schwankungen des Meeres . . 513-528

Erdbeben von Lissabon und zu Visp . . 528

Erdbeben von Lissabon . . . . . . 528-533

Erdbeben in der Schweiz . . . . . 534-543

Erdbeben und vulkanische Wirkungen . . 543-547

Erdbeben und Gasentwicklungen und explosive Gasgemenge. . . . . . . 547-548

Bergschlipfe und Erdbeben. Genetische Verhältnisse 548-603

Mechanische und zersetzende Wirkung des Wassers $548-556$

Erosion, Bergschlipfe und Senkungen . . 556-558

Bergschlipfe und Erdbeben nicht in Ebenen . 558-559

Chemische Wirkungen des Wassers . . . 559-561

Versuche . . . . . . . . . . 561-603

\section{Kapitel LVIII.}

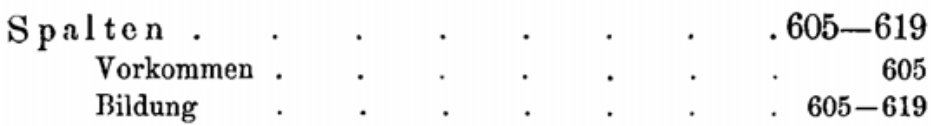

\section{Kapitel hIX.}

Druscnräume . . . . . . . . .

Vorkommen . . . . . . . . . . . 620

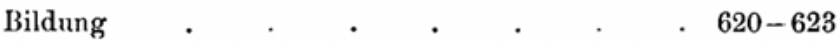

Bildung der Absätze in den Drusenräumen . 623

Eindringende Gewässer . . . . . . 623-624

Wasserdampf in den leeren Räumen der Gebirgsgesteine . . . . . . . . . . 624-626

Kieselige Ueberzüge auf den Wänden der Drusenräume $\dot{\text { der Gewässer in die Hohlräume und }} \cdot \dot{ }$ die Absätze in diesen . . . . . . 628-634

Amorphe und krystallisirte Absätze . . . . 634-636

Die Absätze in Drusenräumen erfordern grofse Zeiträume . . . . . . . . . 636-637

Chemische Prüfungen . $\quad . \quad$. $\quad$. $\quad$. $637-639$

Analysen von Mandelsteinen . . . . . 639-641

Mineralien in Spalten und Klüften der Mandelsteine 641-642

Leichtlösliche Salze nicht in Drusenräumen 642 
Allgemeines über die Bildung der Einschlüsse in den Drusenräumen und Spalten $642-650$

\section{Kapitel Lx.}

Gänge . . . . . . . . . . . . . . .

A. Erzgänge $\quad . \quad$. $\quad . \quad$. $\quad . \quad$. $\quad .652-698$

Structurverhältnisse in den Erzgängen . . 652-654

I. Augit-, Granat-, Kies-, Blendeformation . . 654-655

II. Titanformation . $\quad . \quad$. . . 655

III. Zinn- und Wolframitformation . . . . 655

IV. Quarzformation $\quad . \quad 6 \quad$. $\quad . \quad . \quad 655$

V. Kiesige Blei- und Zinkformation . . . \$55-656

VI. Klinoëdritische Blei- und Zinkformation . 656

VII. Eisenspathformation . . . . . . $\quad 656$

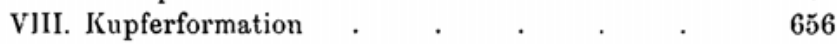

IX. Antimonformation . $\quad . \quad$. $\quad . \quad . \quad 656$

X. Mangan- und Eisenformation . . . 656-657

XI. Jüngere Kobalt- und Nickelformation . $\quad 657$

XII. Flufsspath-, Barytspath-, Blei- und Zinkformation . . . . . . . 657

XIII. Barytspathkupferformation . . . . $\quad 657$

XIV. Silberformation $\quad . \quad$. $\quad . \quad$. $\quad .657-658$

Folgerungen aus dem Zusammenvorkommen verschiedener Gangglieder . . . . . . 658-665

Beziehungen zwischen den Erzgängen und dem

Nebengesteine . . . . . . . $665-673$

Genesis der Erze . . . . . . . . $673-698$

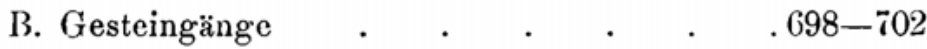

Ausfüllung der Spalten auf mechanischem Wege 699-702

\section{Kapitel IXXI.}

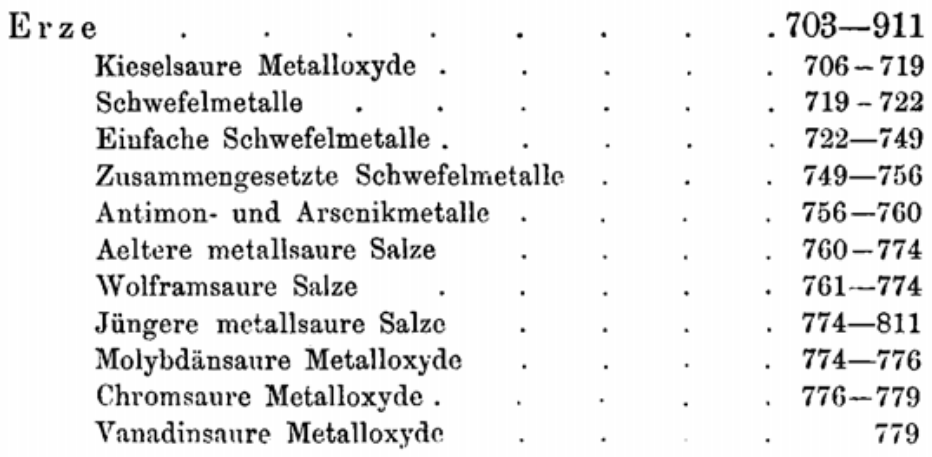


Inhalt.

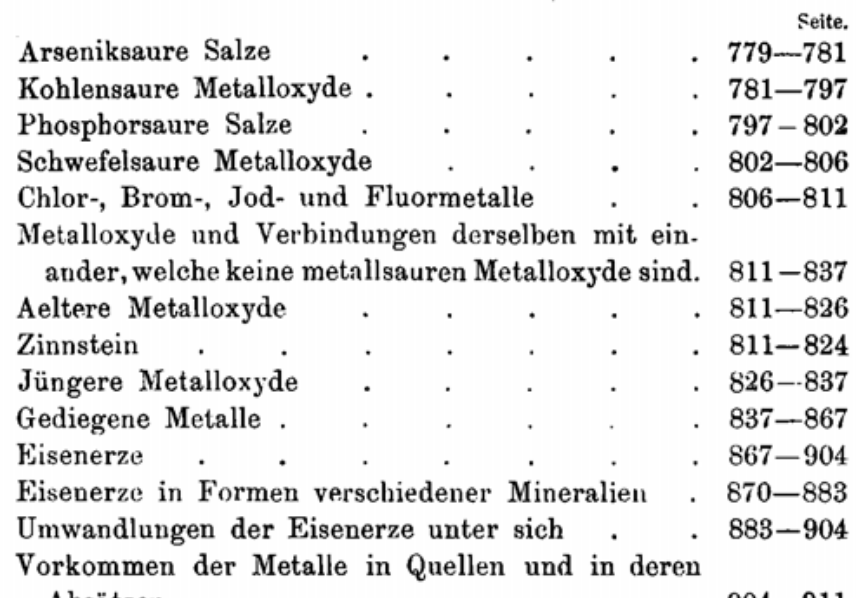
Absätzen
. 904-911 